\title{
Learning About and Destigmatizing Substance Use Disorders: a Video- Based Educational Module Using Simulated Patients
}

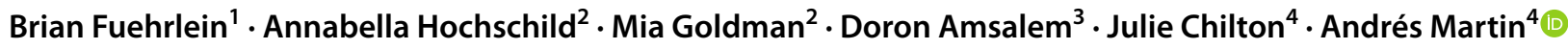

Received: 13 June 2021 / Accepted: 28 October 2021 / Published online: 9 November 2021

(c) Academic Psychiatry 2021

\begin{abstract}
Objective The authors developed and tested video clips depicting three paradigmatic substance use disorder (SUD) clinical scenarios, each portrayed by a different simulated patient interacting with the same clinician.

Methods The authors embedded 21 short video clips (with a cumulative duration of $27 \mathrm{~min}$ ) into a 2-h session on SUDs. The didactic was delivered online through synchronous videoconferencing with Zoom. The primary outcome compared learners' scores on the Attitudes and Confidence in the Treatment of Patients with Substance Use Disorders (ACT-SUDS) before and after participating in the didactic.

Results Fifty-eight second-year medical students participated and completed the survey prior to the didactic; 42 (72\%) of them completed the survey immediately after. Compared to baseline, ACT-SUDS increased after the didactic: from $3.7 \pm$ 0.5 to $4.0 \pm 0.4$ (mean difference $=0.4$ [95\% confidence interval $=0.2-0.5$ ], paired $t=5.75, p<0.001$ ), as did each of its four subscales: confidence, enjoyment, SUD as a medical disorder, and attitudes toward Alcoholics Anonymous (AA; $t \geq$ $3.0, p \leq 0.005)$.

Conclusions The video-based educational module proved easy to implement in the virtual classroom and led to measurable changes in perceptions and attitudes toward SUDs. The module is available to view or freely download and is amenable for adaptation by end-use instructors.
\end{abstract}

Keywords Substance use disorders (SUDs) $\cdot$ Alcoholics/Narcotics Anonymous (AA/NA) $\cdot$ Simulated patients $\cdot$ Didactic $\cdot$ Stigma

Substance use disorders (SUDs) are a major source of morbidity and mortality worldwide. In the USA, nearly a quarter of all deaths are attributed either directly or indirectly to substance use [1]. According to a 2019 national survey, approximately $8 \%$ of individuals age 12 or over had a diagnosable SUD in the past year [2]. Many barriers to treatment persist $[2,3]$, and it is estimated that upwards of $90 \%$ of those needing treatment never receive it [3]. Problematic use of substances in those who do not meet full criteria for a SUD is very common in healthcare settings: it is estimated

Andrés Martin

andres.martin@yale.edu

1 Yale School of Medicine, New Haven, CT, USA

2 Tel Aviv University Faculty of Medicine, Ramat Aviv, Israel

3 Columbia University Vagelos College of Physicians \& Surgeons, New York, NY, USA

4 Yale Child Study Center, New Haven, CT, USA that $20 \%$ of patients in primary care and $60 \%$ in more intensive settings use substances in a problematic way [4]. The fact that nearly all physicians will encounter patients with SUDs and problematic use of substances regularly [5], combined with the barriers to treatment and limited availability of specialists, indicates the importance of proper SUD training for all physicians.

This point was driven home by Ram and Chisolm in 2015 [6], when they studied SUD training deficiencies in US medical schools. On average, medical schools devoted only $12 \mathrm{~h}$ of curricular time to SUDs. Additionally, 119 of 125 schools provided SUD education as part of a larger course, with only 12 offering it as a required course and 45 as an elective [7]. Despite the importance SUDs play in the role of those with mental illness, the Accreditation Council for Graduate Medical Education requires only 1 month of SUD training during 48 months of psychiatry residency training [8], and even less than that for most other specialties, further contributing to deficiencies in clinical care. 
Medical students are particularly vulnerable to negative attitudes toward substance use disorders $[9,10]$. Research also suggests that a physician's attitude and confidence are strongly correlated with screening and treatment practices for patients with SUDs [10]. Negative attitudes, coupled with a lack of confidence, may impact the quality of care provided. We previously developed, implemented, and tested a novel SUD curriculum for medical students [11]. We found that this curriculum had a positive impact on learners' attitudes, confidence, and knowledge. In an effort to enhance this didactic approach and make it easier for other institutions to adopt, we developed a set of complementary video recordings using simulated patients (SPs).

Simulation provides an alternative to traditional didactics and is commonly used as an educational resource. SPs are professional actors trained to portray a specific medical condition or symptom. Training with SPs is effective and correlates with high learner satisfaction. The use of SPs is of particular interest in psychiatry, given the nuances in diagnostic and interpersonal communication so critical to the discipline [12].

We hypothesized that medical students' attitudes, confidence, and knowledge regarding SUDs would increase from baseline after exposure to this new, SP-enhanced education module.

\section{Methods}

Participants were second-year medical students at the New York State/American Program of Tel Aviv University Faculty of Medicine, who completed assessments at two time points: one at the start (time 1) and another after completion of the core curriculum's week-long intensive course in pre-clinical psychiatry (time 2 ). We conducted the module during a 2-h block of dedicated classroom time as an integral component of the course. We incorporated time before and after the session (10 min overall) for students to complete the survey described below. We conducted the session during the COVID-19 pandemic, with content delivered entirely online through the videoconferencing platform Zoom (San Jose, CA). This approach permitted the course to be offered virtually through synchronous content delivery and allowed for effective interaction between faculty and students across different time zones (spanning an 11-h differential).

Before starting data collection, we obtained approval from the Tel Aviv University and Yale School of Medicine Institutional Review Boards (Protocols 0002765-1 and 2000029894, respectively). The study was deemed exempt of review, with completion of the survey representing tacit consent. Participation was voluntary and learners were aware that results of the surveys would not be accessible to faculty responsible for any of their course evaluations. We collected no personally identifying information.

The lead author developed an educational module based on and complementing a previously published curriculum [11]. This module includes video clips of relevant clinical interactions used to exemplify key concepts, with a particular emphasis on psychosocial interventions. The module addresses four main content areas: Introduction to substance use disorders; introduction to alcohol; introduction to opiates; and Alcoholics Anonymous (AA) and Narcotics Anonymous (NA). In the videos, the lead author interacts individually with three different SPs: "Steve," who has relapsed to alcohol use and had prior minimal involvement in AA; "Jim," who has relapsed to alcohol use and has reservations given his previous experience with AA; and "Donna," who has recently developed an opioid use disorder (OUD), including fentanyl use, has learned about the importance of naloxone availability following a friend's fatal overdose, and is considering attending NA for the first time. The three depicted patients are professional actors hired through the Standardized Patient Program of the Teaching and Learning Center at the Yale School of Medicine. The actors are experienced in medical settings and followed accepted best practices for standardized patients [13].

The three source videos and all 22 derivative clips are available online [14].

Participants provided demographic information and completed the Attitudes and Confidence in the Treatment of Patients with Substance Use Disorders (ACT-SUDS) survey [11] before and after the module. The ACT-SUDS is a 19-item survey with good internal consistency (Cronbach $\alpha=0.77$ ) and sensitivity to change. Respondents indicate how strongly they agree or disagree on a five-point Likert scale with positive and negative statements about individuals with SUDs; some of the items are reverse-coded to prevent response acquiescence bias. The scale yields a global score and four subscale scores: Confidence in assessing substance use disorders (5 items); Enjoyment in working with patients with substance use disorders (3 items); Belief that a substance use disorder is a Chronic medical illness (5 items); and Attitudes toward AA (4 items). Next, we asked students to provide the first three words or short phrases "that come to mind when you think of someone with a substance use disorder." Finally, we included five factual questions with forced single response answers of content covered during the session.

Participants completed surveys through their preferred, WiFi-enabled personal devices. We collected information securely through Qualtrics (Provo, UT), and analyzed data using SPSS version 25 (Armonk, NY). We compared differences between the two time points using paired- $t$ or $\mathrm{McNe}$ mar tests for continuous or categorical data, respectively. We used word cloud generator software (wordclouds.com; 
Zygomatic Inc., Vianen, The Netherlands) to visually depict participants' word choices. Word clouds can be easily incorporated into educational activities in order to visualize common underlying themes identified by learners [15].

\section{Results}

All students enrolled in the course ( $n=58,47 \%$ women) were invited to participate. All participants completed the survey before (100\%), and 42 (72\%) after the didactic. Many participants had experiences with substance use disorders: in a friend or relative (57\%), or personally themselves (14\%). Almost one-fourth (23\%) had previously been involved in the care of patients with mental illnesses. Two-thirds of participants considered the possibility of specialization or post-graduate training in addiction medicine.

ACT-SUDS scores increased after the didactic: from 3.7 \pm 0.5 to $4.0 \pm 0.4$ (mean difference $=0.4$ [95\% confidence interval $=0.2-0.5]$, paired $-t=5.75, p<0.001$ ), as did each of its four subscales $(t \geq 3.0, p \leq 0.005$; Table 1$)$. Of note, there was no difference in perceptions about AA across self-assessment categorization as either "spiritual" (48\% of participants) or "religious" (33\%; $p>0.05)$. In addition to attitudes and confidence, factual knowledge increased in three of the five forced-response questions. Fig. 1 provides a visual rendering of all words proffered by participants before and after the didactic.

\section{Discussion}

We found that video clips of clinical interactions between SPs and a psychiatrist with addiction expertise complemented an SUD didactic curriculum, and effectively highlighted psychosocial interventions such as AA/NA. We have created an online repository of our videos, so educators can freely use the clips to synergize and enhance their teaching of SUD content. The videos were easy to implement in the classroom, were well received by learners, led to measurable changes in their attitude and knowledge ratings pertaining to underlying content, and are amenable to adaptation by end-use instructors. Finally, the video-enhanced educational module has the advantage of offering learners a more standardized educational experience.

The module can be edited to address the specific needs of different groups of learners. For example, in this study, we addressed second-year medical students during a 2-h slot in their pre-clinical psychiatry curriculum. To that end, we used all 21 available video clips (with a total running time of $27 \mathrm{~min}$ ). Depending on the specific needs and training level of target learners, the available content can be adjusted accordingly — for example, to emphasize a specific area of SUDs.

Following the module, changes in attitude and confidence were stronger than in factual knowledge. This difference was due to having a standardized instrument for the first two components, but not for the third. Of the two questions with no change between time points, the first was on the number of ounces of 80 -proof $(40 \%)$ ethanol in a standard drink (1.5). The item bumped into a ceiling effect, given the highly accurate response rate at baseline (79\%). The second question, on the opioid use disorder mortality rate after 30 years (50\%), may have simply been too hard for students to believe as factual [16].

As the current opioid crisis continues, clinicians will need to be prepared to treat patients with SUDs in their careers. Additionally, as states move toward the legalization of formerly prohibited substances, thereby potentially increasing the prevalence of SUDs, doctors must be prepared to treat patients utilizing recreational substances in a harmful manner [17]. Moreover, if the COVID-19 pandemic results in the predicted upsurge in mental health conditions, including SUDs, the medical students of today and the physicians of tomorrow will need to be well versed in managing these treatable conditions [18].

At baseline, participants described individuals with SUDs with words of predominantly negative valence: "helpless," "sad," "struggle," "pain," "dependent," "desperate," and "addict" (as opposed to "addiction"). Even though over half of the participants acknowledged having a friend or relative with a SUD, and nearly a quarter
Table 1 Outcomes of videoenriched didactic on attitudes and confidence in the treatment of patients with substance use disorders $(n=42)$

\begin{tabular}{|c|c|c|c|c|c|c|c|}
\hline \multirow[t]{2}{*}{ Aggregate component } & \multicolumn{2}{|l|}{ Pre } & \multicolumn{2}{|l|}{ Post } & \multirow{2}{*}{$\begin{array}{l}\text { Difference } \\
\text { Mean }(95 \% \text { CI })\end{array}$} & \multicolumn{2}{|l|}{ Statistic } \\
\hline & Mean & SD & Mean & SD & & Paired $t_{d f=47}$ & $p$ \\
\hline 1. Confidence & 2.7 & 1.0 & 3.7 & 0.7 & $1.0(0.7,1.3)$ & 6.09 & $<0.001$ \\
\hline 2. Enjoyment & 2.7 & 0.9 & 3.1 & 0.7 & $0.4(0.1,0.6)$ & 3.13 & 0.003 \\
\hline 3. Chronic medical illness & 4.3 & 0.5 & 4.5 & 0.4 & $0.2(0.1,0.4)$ & 3.16 & 0.003 \\
\hline 4. Attitudes toward AA & 3.9 & 0.9 & 4.2 & 0.6 & $0.4(0.1,0.6)$ & 3.00 & 0.005 \\
\hline 5. Overall & 3.7 & 0.5 & 4.0 & 0.4 & $0.4(0.2,0.5)$ & 5.75 & $<0.001$ \\
\hline
\end{tabular}

Means of Likert scale: 1 = strongly disagree; 2 = disagree; $3=$ neutral; $4=$ agree; $5=$ strongly agree. Scale and aggregate components based on Feeley et al. [11]. 

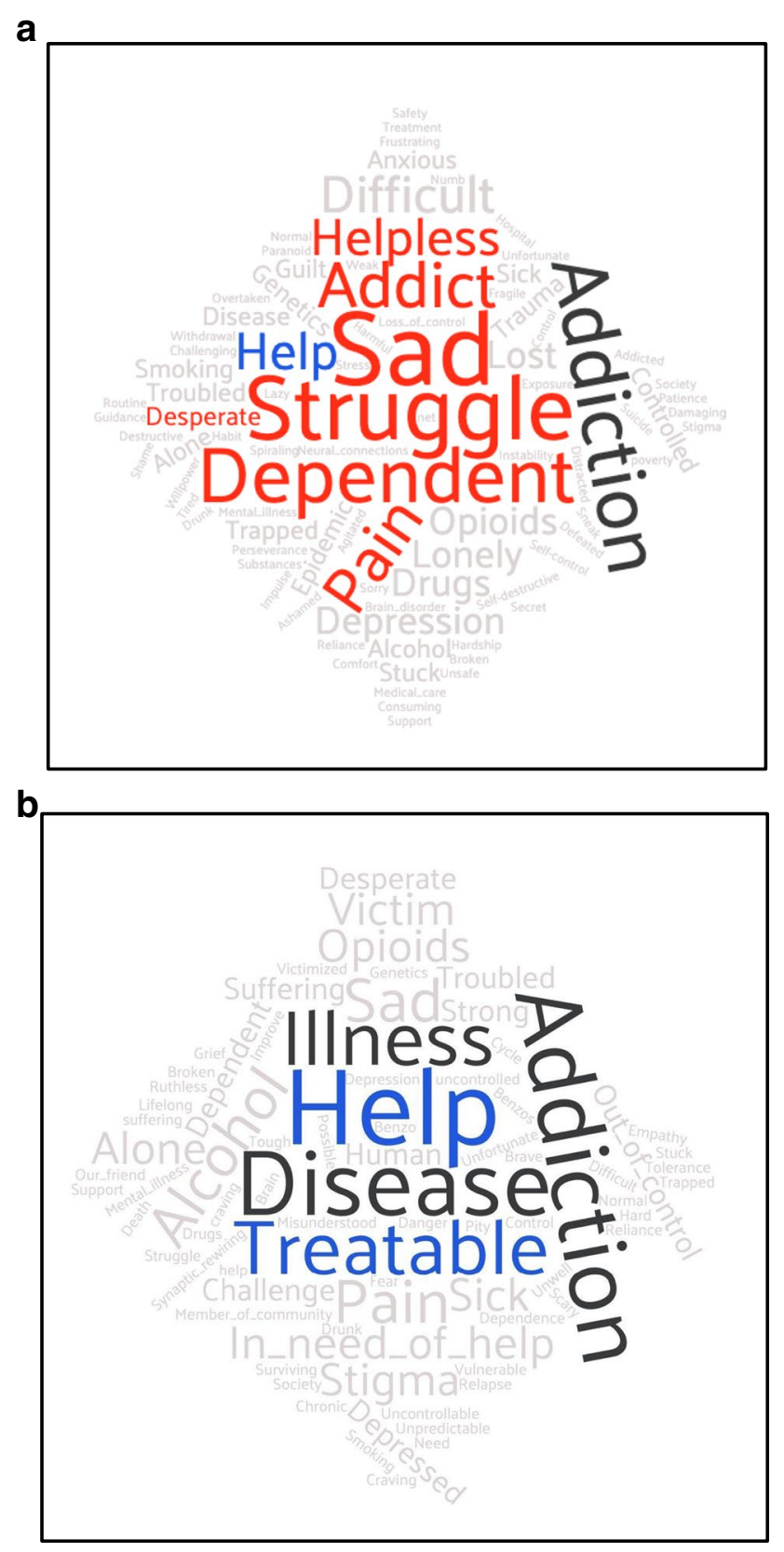

Fig. 1 Word clouds based on the prompt "provide the first three words or short phrases that come to mind when you think of someone with a substance use disorder." A Time 1 ( $n=174$ words); B time 2 ( $n=151$ words). Word sizes are proportional to the frequency of their occurrence.

had been involved in the treatment of patients with SUD, the majority still gravitated to non-medical descriptors of those with a SUD, implying a negative value judgment. The attribution of personal or moral failings to those with a disease is not new in medicine, particularly for disease processes that are not well understood, for which the prognosis is poor, or where behavioral choices may be attributed to those afflicted: for example, the view of AIDS in the latter part of the twentieth century. Following the module, the words chosen by participants were notably different. No negative terms were used, and neutral descriptors increased in frequency: "addiction" (as opposed to "addict"); "illness"; and "disease." At least two terms with a positive valence emerged after the session: "help" and "treatable."

Prior to the module, participants held predominantly negative views of those with SUDs. By both quantitative and qualitative metrics, the 2-h module led to a cognitive reappraisal, in which SUDs were legitimized as medical conditions. The pre-existing knowledge regarding SUDs was largely inconsistent with current medical knowledge [19]. However, it was certainly in keeping with the stigmatized views that still prevail [20]. The video-based component may have had a positive impact raising awareness and destigmatizing individuals with SUDs. This effect may be particularly beneficial for students who will not go on to pursue careers in mental health or addiction medicine, as they may have more entrenched negative or dismissive views.

We note six main limitations to our study. First, our emphasis was largely on psychosocial interventions like AA/NA, with relatively small attention to other aspects of SUD care, such as medication-assisted treatment. Second, we measured attitudes and knowledge as outcomes of interest, but the favorable changes we found may not necessarily lead to behavioral change, such as better care for patients with SUDs. This consideration is especially relevant to second-year medical students, who may not get to care independently for such patients until 2 or 3 years later. Additional experience and direct contact with individuals with SUDs would certainly complement this video-based exposure to such an important clinical population. Third, given the short time interval between the two assessment points and the brief overall duration of the course, we cannot rule out the role of immediacy and social desirability biases. Fourth, with its pre-post design, our study did not include a comparator or evaluate the incremental and unique contribution of the video content, above and beyond that of the didactic component alone. Fifth, our sample size was modest. Finally, even though generalizability from a single instructor and medical school class cannot be assumed, we encourage end users to adjust our materials to learners from other health professions commonly involved in the care of patients with SUDs (e.g., nursing, social work).

Acknowledgements We appreciate the professional actors' contributions and the learners' engaged participation. We are grateful to Barbara Hildebrand of the Standardized Participant Program, Teaching and Learning Center, Yale School of Medicine.

Funding Supported by the Riva Ariella Ritvo Endowment at the Yale School of Medicine, and by NIMH R25 MH077823, "Research Education for Future Physician-Scientists in Child Psychiatry." 


\section{Declarations}

Ethical Considerations The Yale Human Investigations Committee (Protocol \# 2000029894) and the Ethics Committee of Tel-Aviv University (Protocol \# 0002765-1) approved the study and deemed it exempt, with survey completion standing for tacit consent.

Disclosures On behalf of all authors, the corresponding author states that there is no conflict of interest.

\section{References}

1. Mokdad AH, Marks JS, Stroup DF, Gerberding JL. Actual causes of death in the United States, 2000. JAMA. 2004;291:1238-45 Available from: https://www.ncbi.nlm.nih.gov/pubmed/15010446.

2. McKance-Katz E National Survey on Drug Use and Health: detailed tables. Subst Abus Ment Heal Serv Adm [SAMHSA]. 2017;1-63. Available from: https://www.samhsa.gov/data/sites/ default/files/reports/rpt31102/2019NSDUH-Women/Women 2019 NSDUH.pdf. Accessed 6 Jun 2021.

3. Jackson A, Shannon L. Barriers to receiving substance abuse treatment among rural pregnant women in Kentucky. Matern Child Health J. 2012;16:1762-70 Available from: 2011/12/06 https:// www.ncbi.nlm.nih.gov/pubmed/22139045.

4. Saitz R, Horton NJ, Sullivan LM, Moskowitz MA, Samet JH. Addressing alcohol problems in primary care: a cluster randomized, controlled trial of a systems intervention. The screening and intervention in primary care (SIP) study. Ann Intern Med. 2003;138:372-82 Available from: https://www.ncbi.nlm.nih.gov/ pubmed/12614089.

5. McLellan AT, Woodworth AM. The affordable care act and treatment for "substance use disorders:" implications of ending segregated behavioral healthcare. J Subst Abus Treat. 2014;46:541-5 Available from: https://www.ncbi.nlm.nih.gov/pubmed/24679908.

6. Ram A, Chisolm MS. The time is now: improving substance abuse training in medical schools. Acad Psychiatry. 2016;40:454-60 2015/03/10 Available from: https://www.ncbi.nlm.nih.gov/pub$\mathrm{med} / 25749922$.

7. Yoast RA, Filstead WJ, Wilford BB, Hayashi S, Reenan J, Epstein J. Teaching about substance abuse. Virtual Mentor. 2008;10:21-9 Available from: https://www.ncbi.nlm.nih.gov/pubmed/23206739.

8. Accreditation Council for Graduate Medical Education: ACGME (2020) program requirements for graduate medical education in psychiatry (effective July 2020). Available from: https://www. acgme.org. Accessed 1 Jun 2021.

9. Roche AM, Parle MD, Stubbs JM, Hall W, Saunders JB. Management and treatment efficacy of drug and alcohol problems: what do doctors believe? Addiction. 1995;90:1357-66 Available from: https://www.ncbi.nlm.nih.gov/pubmed/8616464.
10. Geller G, Levine DM, Mamon JA, Moore RD, Bone LR, Stokes EJ. Knowledge, attitudes, and reported practices of medical students and house staff regarding the diagnosis and treatment of alcoholism. JAMA. 1989;261:3115-20 Available from: https:// www.ncbi.nlm.nih.gov/pubmed/2716143.

11. Feeley RJ, Moore DT, Wilkins K, Fuehrlein B. A focused addiction curriculum and its impact on student knowledge, attitudes, and confidence in the treatment of patients with substance use. Acad Psychiatry. 2018;42:304-8 2017/08/05. Available from: https://www.ncbi.nlm.nih.gov/pubmed/28776146.

12. Piot M-A, Attoe C, Billon G, Cross S, Rethans J-J, Falissard B. Simulation training in psychiatry for medical education: a review. Front Psychiatry. 2021;12:1-12.

13. Lewis KL, Bohnert CA, Gammon WL, Hölzer H, Lyman L, Smith C, et al. The Association of Standardized Patient Educators (ASPE) Standards of Best Practice (SOBP). Adv Simul. 2017;2:1-8.

14. Figshare [Internet]. Available from: https://figshare.com/s/618f9 4116cc198ce90e4

15. DePaolo CA, Wilkinson K. Get your head into the clouds: using word clouds for analyzing qualitative assessment data. TechTrends. 2014;58:38-44.

16. Hser YI. Predicting long-term stable recovery from heroin addiction: findings from a 33-year follow-up study. J Addict Dis. 2007;26:51-60 Available from: https://www.ncbi.nlm.nih.gov/ pubmed/17439868.

17. Zvonarev V, Fatuki TA, Tregubenko P. The public health concerns of marijuana legalization: an overview of current trends. Cureus. 2019;11:e5806 Available from: https://www.ncbi.nlm.nih.gov/ pubmed/31723545.

18. Becker WC, Fiellin DA. When epidemics collide: coronavirus disease 2019 (COVID-19) and the opioid crisis. Ann Intern Med. 2020;173:59-60 Available from: https://www.ncbi.nlm.nih.gov/ pubmed/32240291.

19. Nyblade L, Stockton MA, Giger K, Bond V, Ekstrand ML, Lean RM, et al. Stigma in health facilities: why it matters and how we can change it. BMC Med. 2019;17:25 Available from: https:// www.ncbi.nlm.nih.gov/pubmed/30764806.

20. Smith LR, Earnshaw VA, Copenhaver MM, Cunningham CO. Substance use stigma: reliability and validity of a theory-based scale for substance-using populations. Drug Alcohol Depend. 2016;162:34-43 Available from: https://www.ncbi.nlm.nih.gov/ pubmed/26972790.

Publisher's Note Springer Nature remains neutral with regard to jurisdictional claims in published maps and institutional affiliations. 